\title{
Leptonic $C P$ and flavor violations in SUSY GUT with right-handed neutrinos
}

\author{
Kaigo Hirao (1) and Takeo Moroi \\ Department of Physics, University of Tokyo, Tokyo 113-0033, Japan
}

(Received 19 February 2021; accepted 17 July 2021; published 30 August 2021)

\begin{abstract}
We study leptonic $C P$ and flavor violations in supersymmetric grand unified theory with right-handed neutrinos, paying attention to the renormalization group effects on the slepton mass matrices due to the neutrino and grand unified theory Yukawa interactions. In particular, we study in detail the impacts of the so-called Casas-Ibarra parameters on $C P$ and flavor violating observables. The renormalization group effects induce $C P$ and flavor violating elements of the supersymmetric breaking scalar mass squared matrices, which may result in sizable leptonic $C P$ and flavor violating signals. Assuming a seesaw formula for the active neutrino masses, the renormalization group effects have been often thought to be negligible as the right-handed neutrino masses become small. With the most general form of the neutrino Yukawa matrix, i.e., taking into account the Casas-Ibarra parameters; however, this is not the case. We found that the maximal possible sizes of signals of leptonic $C P$ and flavor violating processes are found to be insensitive to the mass scale of the right-handed neutrinos and that they are as large as (or larger than) the present experimental bounds irrespective of the right-handed neutrino masses.
\end{abstract}

DOI: $10.1103 /$ PhysRevD.104.035038

\section{INTRODUCTION}

Even though the standard model (SM) of particle physics successfully explains many of results of high energy experiments, the existence of a physics beyond the SM (BSM) has been highly anticipated. Particularly, from a particle cosmology point of view, there are many mysteries that cannot be explained in the framework of the SM, like the existence of dark matter, the origin of the baryon asymmetry of the Universe, the dynamics of inflation, and so on. Many experimental efforts have been performed to find signals of the BSM physics.

In the search of the BSM signals, energy and precision frontier experiments are both important. The energy frontier experiments, represented by collider experiments like the LHC at present, may directly find and study particles in BSM models, but their discovery reach is limited by the beam energy. On the contrary, the precision frontier ones may reach the BSM whose energy scale is much higher than the energy scale of the LHC experiment, although information about the BSM from those experiments may be indirect. Currently, the LHC has not found any convincing evidence of the BSM physics. In such a circumstance, it is important to reconsider the role of precision frontier

Published by the American Physical Society under the terms of the Creative Commons Attribution 4.0 International license. Further distribution of this work must maintain attribution to the author(s) and the published article's title, journal citation, and DOI. Funded by SCOAP ${ }^{3}$. experiments and study what kind of signal may be obtained from them.

In this paper, we study $C P$ and flavor violations in models with supersymmetry (SUSY), and their impacts on ongoing and future experiments. Even though the LHC has not found any signal of SUSY particles with their mass scale of $\sim \mathrm{TeV}$, the SUSY is still a well-motivated candidate of BSM physics. Taking into account the observed Higgs mass of $125.10 \pm 0.14 \mathrm{GeV}$ [1], heavy SUSY particles (more specifically, heavy stops) are preferred to push up the Higgs mass via radiative corrections [2-5]. We note that, in a large class of models, SUSY particles can acquire masses of $\sim O(10-100) \mathrm{TeV}$ [6-10]. In SUSY grand unified theory (GUT) models, such heavy SUSY particles (in particular, heavy sfermions) help suppressing the rate of the proton decay mediated by dimension-five baryon- and leptonnumber violating operators $[11,12] .{ }^{1}$ Here, we pay particular attention to SUSY SU(5) GUT with right-handed neutrinos, in which superparticle masses are much above the $\mathrm{TeV}$ scale, because (i) with the particle content of the minimal SUSY standard model, the gauge coupling unification at $M_{\mathrm{GUT}} \sim O\left(10^{16}\right) \mathrm{GeV}$ is suggested, and also because (ii) right-handed neutrinos are well motivated to explain the origin of the active neutrino masses via the seesaw mechanism [13-15]. In SUSY GUT with righthanded neutrinos, some of the cosmological mysteries

\footnotetext{
${ }^{1}$ The decay rate of the proton via the dimension-five operators is highly model dependent and we do not consider the detail about the proton decay in the present analysis.
} 
mentioned above may be also solved; the baryon asymmetry of the Universe may be explained by the leptogenesis scenario [16], while the lightest superparticle may play the role of dark matter. Compared to the SM, the SUSY models contain various new sources of $C P$ and flavor violations. It may cause significant $C P$ and flavor violating processes which cannot be explained in the SM. If such processes are experimentally observed, they can be smoking gun evidences of the BSM physics, based on which we may study the BSM model behind the $C P$ and flavor violations.

It has been well known that the renormalization group effect may induce $C P$ and flavor violating off-diagonal elements of the slepton mass matrices [17-21]; in the framework of our interest, the left- and right-handed slepton mass matrices are affected by the renormalization group effects from the neutrino Yukawa coupling and the running above the GUT scale $M_{\mathrm{GUT}}$, respectively, even though the lepton flavor is conserved in the Yukawa interaction of the minimal SUSY standard model (MSSM). Thus, even though the slepton mass matrices are universal at some high scale (for example, Planck scale), such universalities are violated by the renormalization group effects. The effects of the offdiagonal elements of the slepton mass matrices on $C P$ and/or flavor violating observables have been studied (see, for example, [22-69]). In particular, it has been pointed out that, even if the MSSM particles are out of the reach of the LHC experiment, the signal of the MSSM may be observed by ongoing or future $C P$ or flavor violation experiments. In previous studies, the neutrino Yukawa matrix was reconstructed by combining the seesaw formula with the active neutrino mass squared differences suggested by the neutrino oscillation experiments. Then, the neutrino Yukawa coupling constants are inversely proportional to the square root of the mass scale of the right-handed neutrinos. With adopting simple assumption about the neutrino sector, i.e., the universal masses for the right-handed neutrinos as well as a simple mixing structure, the renormalization group effects due to the neutrino Yukawa couplings become irrelevant as the mass scale of the right-handed neutrinos becomes smaller. However, as pointed out by Casas and Ibarra (CI) [39], there exist several parameters (which we call CI parameters) that complicate the mixing structure of the neutrino Yukawa matrix.

In this paper, we study $C P$ and flavor violating processes paying particular attention to the effects of the CI parameters, as well as the effects of the nonuniversality of the right-handed neutrino masses, whose effects have not been fully investigated so far. (For some discussion about the effect of the CI parameters, see $[32,43,44,46,47$, $52-56,61,67,68])$. The organization of this paper is as follows. In Sec. II, we introduce the model based on which we perform our analysis. In Sec. III, we show the results of our numerical analysis. Section IV is devoted to conclusions and discussion.

\section{MODEL AND PARAMETRIZATION}

In this section, we introduce the model we consider. We also summarize our convention of the model parameters, including CI parameters and GUT phases. To this end, we define the couplings and specify the flavor basis we use for each effective theories and explain how they are related at the matching scales.

Effective theories in our model appropriate for the each energy scales lower than $M_{\mathrm{Pl}}$ are shown in Fig. 1, where

(i) QEDQCD: QED and QCD.

(ii) MSSMNR: MSSM with three generations of righthanded neutrinos. (Here, NR indicates a theory with right-handed neutrinos.)

(iii) SU(5)NR: minimal SU(5) GUT with three generations of right-handed neutrinos.

At each renormalization scale $Q$, we use the relevant effective theory as we explain below.

We assume that the effect of the SUSY breaking is mediated to the visible sector (containing the MSSM particles and right-handed neutrinos) at the reduced Planck scale $M_{\mathrm{Pl}} \simeq 2.4 \times 10^{18} \mathrm{GeV}$. Then, at the scales between $M_{\mathrm{Pl}}$ and $M_{\mathrm{GUT}}$, the model is described by SU(5)NR. In order to introduce three generations of quarks and leptons, three copies of chiral supermultiplets $\Phi_{i}$ and $\Psi_{i}$, which are in he $\overline{\mathbf{5}}$ and $\mathbf{1 0}$ representations of SU(5), respectively, are introduced. (Here, the $i=1-3$ is the generation index.) As the conventional SU(5) GUT, $\Phi_{i}$ is composed of the righthanded down-type quark multiplets $\bar{D}_{i}$ and the lepton doublets $L_{i}$, while $\Psi_{i}$ is composed of the quark doublets $Q_{i}$, the right-handed up-type quark multiplets $U_{i}$, and the right-handed charged lepton $\bar{E}_{i}$. The right-handed neutrinos $\bar{N}_{i}$ of MSSMNR are added as SU(5) singlets $\Upsilon_{i}$. The MSSM Higgs doublets $H_{u}$ and $H_{d}$ are embedded into $H$ and $\bar{H}$, which are $\mathrm{SU}(5) \mathbf{5}$ and $\overline{\mathbf{5}}$ representations, respectively. There is also a multiplet that breaks $\mathrm{SU}(5)$ symmetry to the SM gauge group. We assume that a chiral multiplet in the adjoint representation of $\mathrm{SU}(5)$, which we call $\Sigma$, is responsible for the breaking of the $\mathrm{SU}(5)$ symmetry. The vacuum expectation value (VEV) of $\Sigma$ is denoted as $\langle\Sigma\rangle=\operatorname{diag}\left(2 v_{\mathrm{GUT}}, 2 v_{\mathrm{GUT}}, 2 v_{\mathrm{GUT}},-3 v_{\mathrm{GUT}},-3 v_{\mathrm{GUT}}\right)$.

We consider the superpotential of SU(5)NR in the following form:

$$
\begin{aligned}
& W_{\mathrm{SU}(5) \mathrm{NR}}=W_{\mathrm{SU}(5) \mathrm{NR}}^{\mathrm{ren}}+W_{\mathrm{SU}(5) \mathrm{NR}}^{\text {nonren }}, \\
& W_{\mathrm{SU}(5) \mathrm{NR}}^{\mathrm{ren}}=W_{\mathrm{SU}(5) \mathrm{NR}}^{\mathrm{matter}}+W_{\mathrm{SU}(5) \mathrm{NR}}^{\mathrm{Higg}},
\end{aligned}
$$

\begin{tabular}{cccccccc} 
QEDQCD & & SM & & MSSM & & MSSMNR & SU(5)NR \\
\hline & $M_{t}$ & & $M_{S}$ & & $\underline{M}_{N_{1,2,3}}^{\prime}$ & $M_{\mathrm{GUT}}^{\prime}$ & $M_{\mathrm{Pl}}$
\end{tabular}

FIG. 1. The effective theories used for each regions of the renormalization scale $Q$. 
and

$$
\begin{aligned}
W_{\mathrm{SU}(5) \mathrm{NR}}^{\mathrm{matter}}= & \frac{1}{4}\left(f_{u}\right)_{i j} \Psi_{i} \Psi_{j} H+\sqrt{2}\left(f_{d}\right)_{i j} \Psi_{i} \Phi_{j} \bar{H} \\
& +\left(f_{\nu}\right)_{i j} \Upsilon_{i} \Phi_{j} H+\frac{1}{2}\left(M_{\Upsilon}\right)_{i j} \Upsilon_{i} \Upsilon_{j},
\end{aligned}
$$

where $f_{u}, f_{d}$, and $f_{\nu}$ are $3 \times 3$ coupling matrices while $M_{\Upsilon}$ is a $3 \times 3$ matrix with mass dimension one. Notice that $f_{u}$ and $M_{\Upsilon}$ are symmetric. In Eq. (2.3), the summations over SU(5) indices are implicit. (We follow [60] for the group theoretical notations.) $W_{\mathrm{SU}(5) \mathrm{NR}}$ consists of the renormalizable part $W_{\mathrm{SU}(5) \mathrm{NR}}^{\mathrm{ren}}$ and the nonrenormalizable part $W_{\mathrm{SU}(5) \mathrm{NR}}^{\mathrm{nnonre}}$. $W_{\mathrm{SU}(5) \mathrm{NR}}^{\text {ren }}$ is further split into $W_{\mathrm{SU}(5) \mathrm{NR}}^{\text {matter }}$ (i.e., the superpotential containing the matter sector) and $W_{\mathrm{SU}(5) \mathrm{NR}}^{\mathrm{Higgs}}$ (i.e., the superpotential for the Higgs sector); $W_{\mathrm{SU}(5) \mathrm{NR}}^{\mathrm{Higs}}$ is the superpotential containing only the Higgs field and $\Sigma$. In Eq. (2.3), $W_{\mathrm{SU}(5) \mathrm{NR}}^{\mathrm{matter}}$ contains superpotential responsible for the up-type, down-type, and neutrino-type Yukawa terms in the MSSMNR. In addition, in order to explain the unification of the down-type and electron-type Yukawa matrices, we assume that $W_{\mathrm{SU}(5) \mathrm{NR}}^{\text {nonen }}$ contains a term in the following form:

$$
W_{\mathrm{SU}(5) \mathrm{NR}}^{\mathrm{nonren}} \ni \frac{\sqrt{2}}{M_{\mathrm{Pl}}} c_{i j} \Psi_{i} \Sigma \Phi_{j} \bar{H} .
$$

Unitary rotations on the family indices can make the coupling matrices to the following forms:

$$
\begin{gathered}
f_{u}=V^{T} \hat{f}_{u} \hat{\Theta}_{q} V, \\
f_{d}=\hat{f}_{d}, \\
f_{\nu}=\tilde{W}^{\dagger} \hat{f}_{\nu} U^{\dagger} \hat{\Theta}_{l}, \\
M_{\Upsilon}=\hat{M}_{\Upsilon},
\end{gathered}
$$

where $\hat{f}_{u}, \hat{f}_{d}, \hat{f}_{\nu}$, and $\hat{M}_{\Upsilon}$ are real diagonal matrices. ${ }^{2}$ In addition, $V$ and $U$ are unitary matrices with only a single $C P$ phase and three mixing angles while $\tilde{W}$ is a general unitary matrix with additional five phases. ${ }^{3}$ Furthermore,

\footnotetext{
${ }^{2}$ The hat on matrix symbols indicates that they are diagonal.

${ }^{3}$ In general, a unitary matrix $\tilde{X}$ can be decomposed as

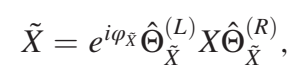

where $\varphi_{\tilde{X}}$ is the overall phase of the matrix $\tilde{X}, \hat{\Theta}_{\tilde{X}}^{(L)}$ and $\hat{\Theta}_{\tilde{X}}^{(R)}$ are diagonal phase matrices parametrized by two physical phases, and $X$ is a unitary matrix parametrized by three mixing angles, $\vartheta_{12}, \vartheta_{13}$, and $\vartheta_{23}$, and a single phase $\delta$ as

$$
X=\left(\begin{array}{ccc}
c_{12} c_{13} & s_{12} c_{13} & s_{13} e^{-i \delta} \\
-s_{12} c_{23}-c_{12} s_{23} s_{13} e^{i \delta} & c_{12} c_{23}-s_{12} s_{23} s_{13} e^{i \delta} & s_{23} c_{13} \\
s_{12} s_{23}-c_{12} c_{23} s_{13} e^{i \delta} & -c_{12} c_{23}-s_{12} c_{23} s_{13} e^{i \delta} & c_{23} c_{13}
\end{array}\right),
$$

with $c_{i j}=\cos \vartheta_{i j}$ and $s_{i j}=\sin \vartheta_{i j}$.
$\hat{\Theta}_{q}$ and $\hat{\Theta}_{l}$ are diagonal phase matrices and represent $C P$ phases intrinsic in SU(5) GUT. Notice that the overall phases of $\hat{\Theta}_{q}$ and $\hat{\Theta}_{l}$ are unphysical because they can be absorbed to $\tilde{W}$. Thus, each of $\hat{\Theta}_{q}$ and $\hat{\Theta}_{l}$ contains two parameters; we parametrize these matrices as

$$
\hat{\Theta}_{f}=\operatorname{diag}\left(1, e^{i \varphi_{f_{2}}}, e^{i \varphi_{f_{3}}}\right),
$$

with $f=q, l$.

In the following argument, we take the flavor basis in which the coupling matrices of SU(5)NR take the forms of Eqs. (2.5)-(2.8) at $Q=M_{\mathrm{GUT}}$. In our discussion, the higher dimensional operator proportional to $c$ is introduced just to guarantee the unification of $\bar{U}_{i}$ and $L_{i}$ into $\overline{\mathbf{5}}$ multiplets of $\mathrm{SU}(5)$. For simplicity, we assume that $c$ is real and diagonal at $Q=M_{\mathrm{GUT}}$ in this basis: $c=\hat{c}$.

At the GUT scale $M_{\mathrm{GUT}}$, SU(5)NR couplings are matched to MSSMNR couplings. The matter sector superpotential of MSSMNR is given in the following form:

$$
\begin{aligned}
W_{\mathrm{MSSMNR}}^{\text {matter }}= & \left(y_{u}\right)_{i j} H_{u} \bar{U}_{i} Q_{j}+\left(y_{d}\right)_{i j} H_{d} \bar{D}_{i} Q_{j} \\
& +\left(y_{e}\right)_{i j} H_{d} \bar{E}_{i} L_{j}+\left(y_{\nu}\right)_{i j} H_{u} \bar{N}_{i} L_{j} \\
& +\frac{1}{2}\left(M_{N}\right)_{i j} \bar{N}_{i} \bar{N}_{j},
\end{aligned}
$$

where $y_{u}, y_{d}, y_{e}$, and $y_{\nu}$ are the MSSMNR Yukawa matrices while $M_{N}$ is the Majorana mass matrix of $\bar{N}$. The MSSMNR chiral multiplets are embedded into the SU(5) NR ones as follows:

$$
\begin{gathered}
\Psi_{i}=\left(\mathcal{U}_{Q}^{\dagger} Q, V^{\dagger} \hat{\Theta}_{q}^{*} \mathcal{U}_{\bar{U}}^{\dagger} \bar{U}, \hat{\Theta}_{l} \mathcal{U}_{\bar{E}}^{\dagger} \bar{E}\right)_{i}, \\
\Phi_{i}=\left(\mathcal{U}_{\bar{D}}^{\dagger} \bar{D}, \hat{\Theta}_{l}^{*} \mathcal{U}_{L}^{\dagger} L\right)_{i}, \\
\Upsilon_{i}=\left(\mathcal{U}_{\bar{N}}^{\dagger} \bar{N}\right)_{i},
\end{gathered}
$$

where $\mathcal{U}_{Q}, \mathcal{U}_{\bar{U}}, \mathcal{U}_{\bar{D}}, \mathcal{U}_{L}, \mathcal{U}_{\bar{E}}$, and $\mathcal{U}_{\bar{N}}$ are $3 \times 3$ unitary matrices that depend on the choice of the flavor basis in the MSSMNR. At the tree level, the matching conditions at $Q=M_{\mathrm{GUT}}$ are obtained as

$$
\begin{gathered}
\mathcal{U}_{\bar{U}}^{T}\left[y_{u}\right]_{Q=M_{\mathrm{GUT}}} \mathcal{U}_{Q}=\left[\hat{f}_{u} V\right]_{Q=M_{\mathrm{GUT}}}, \\
\mathcal{U}_{\bar{D}}^{T}\left[y_{d}\right]_{Q=M_{\mathrm{GUT}}} \mathcal{U}_{Q}=\left[\hat{f}_{d}+\frac{2 v_{\mathrm{GUT}}}{M_{\mathrm{Pl}}} \hat{c}\right]_{Q=M_{\mathrm{GUT}}}, \\
\mathcal{U}_{\bar{E}}^{T}\left[y_{e}\right]_{Q=M_{\mathrm{GUT}}} \mathcal{U}_{L}=\left[\hat{f}_{d}-\frac{3 v_{\mathrm{GUT}}}{M_{\mathrm{Pl}}} \hat{c}\right]_{Q=M_{\mathrm{GUT}}}, \\
\mathcal{U}_{\bar{N}}^{T}\left[y_{\nu}\right]_{Q=M_{\mathrm{GUT}}} \mathcal{U}_{L}=\left[\tilde{W}^{\dagger} \hat{f}_{\nu} U^{\dagger}\right]_{Q=M_{\mathrm{GUT}}}, \\
\mathcal{U}_{\bar{N}}^{T}\left[M_{N}\right]_{Q=M_{\mathrm{GUT}}} \mathcal{U}_{\bar{N}}=\left[\hat{M}_{\Upsilon}\right]_{Q=M_{\mathrm{GUT}}},
\end{gathered}
$$


TABLE I. The Neutrino mixing angles, $C P$ violating phase, mass-squared differences, and the lightest neutrino mass used in our numerical analysis [1]. For the left-handed neutrino mass spectrum, we consider both normal ordering (NO) and inverted ordering (IO) cases.

\begin{tabular}{lcccccc}
\hline \hline $\sin ^{2} \theta_{12}$ & $\sin ^{2} \theta_{23}$ & $\sin ^{2} \theta_{13}$ & $\delta_{\mathrm{CP}}$ & $\Delta m_{31}^{2}\left[\mathrm{eV}^{2}\right]$ & $\Delta m_{21}^{2}\left[\mathrm{eV}^{2}\right]$ & $m_{\nu_{1}}^{2}\left[\mathrm{eV}^{2}\right]$ \\
\hline 0.307 & 0.545 & 0.0218 & $1.36 \pi$ & $+2.453 \times 10^{-3}$ & $7.53 \times 10^{-5}$ & $1.00 \times 10^{-6}$ \\
\hline \hline & & & & $\mathrm{IO}$ & & \\
\hline $\sin ^{2} \theta_{12}$ & $\sin ^{2} \theta_{23}$ & $\sin ^{2} \theta_{13}$ & $\delta_{\mathrm{CP}}$ & $\Delta m_{31}^{2}\left[\mathrm{eV}^{2}\right]$ & $\Delta m_{21}^{2}\left[\mathrm{eV}^{2}\right]$ & $m_{\nu_{3}}^{2}\left[\mathrm{eV}^{2}\right]$ \\
\hline 0.307 & 0.547 & 0.0218 & $1.36 \pi$ & $-2.546 \times 10^{-3}$ & $7.53 \times 10^{-5}$ & $1.00 \times 10^{-6}$ \\
\hline \hline
\end{tabular}

Based on the above relations, the coupling matrices of the SU(5)NR are determined from those of the MSSMNR in our numerical analysis with properly choosing the unitary matrices $\mathcal{U}_{Q}, \mathcal{U}_{\bar{U}}, \mathcal{U}_{\bar{D}}, \mathcal{U}_{L}, \mathcal{U}_{\bar{E}}$, and $\mathcal{U}_{\bar{N}}$. The phase matrices $\hat{\Theta}_{q}$ and $\hat{\Theta}_{l}$, as well as the overall phase of the unitary matrix $\tilde{W}$, can be absorbed into the definitions of the MSSMNR superfields and can be removed from the MSSMNR superpotential. (However, they are physical in the SU(5) NR, and we call them "GUT phases.") Notice that the GUT phases cannot be determined from the structure of the Yukawa matrices in the MSSMNR; in other words, for any values of the GUT phases, the Yukawa matrices in the MSSMNR consistent with observations can be obtained.

Now, let us consider the neutrino masses. For this purpose, it is more convenient to use the flavor basis in which $\bar{N}_{i}(i=1-3)$ become the mass eigenstates (see below). In general, the masses of right-handed neutrinos are different. We denote the mass of $i$ th right-handed neutrino as $\underline{M}_{N_{i}}$, with $\underline{M}_{N_{1}} \leq \underline{M}_{N_{2}} \leq \underline{M}_{N_{3}}$. The dimension-five operator responsible for the Majorana mass terms of the left-handed neutrinos is generated with seesaw mechanism by integrating out right-handed neutrinos [13-15]. Let us define the following diagonal matrix: $\underline{\hat{M}}_{N} \equiv \operatorname{diag}\left(\underline{M}_{N_{1}}, \underline{M}_{N_{2}}, \underline{M}_{N_{3}}\right)$. Using $\underline{\hat{M}}_{N}$, the active neutrino mass matrix is given by ${ }^{4}$

$$
m_{\nu}=v_{u}^{2} \underline{y}_{\nu}^{T} \hat{\underline{M}}_{N}^{-1} \underline{y}_{\nu},
$$

where $v_{u}$ is the VEV of $H_{u}$. In addition, $\underline{y}_{\nu, i j} \equiv y_{\nu, i j}\left(Q=\underline{M}_{N_{i}}\right)$, with $j=1-3$, is the neutrino Yukawa coupling constant at $Q=\underline{M}_{N_{i}}$, where, again, we are adopting the flavor basis in which $\bar{N}_{i}$ is the mass eigenstate.

At the energy scales lower than $\underline{M}_{N_{1}}$, the model is described by the MSSM and one can always work in the basis in which $y_{e}$ is diagonal. In such a basis, $m_{\nu}$ takes the following form.

\footnotetext{
${ }^{4}$ In Eq. (2.19), the running of the Wilson coefficients of the dimension-five operator is neglected in our analysis.
}

$$
m_{\nu}=U_{\mathrm{PMNS}}^{*} \hat{m}_{\nu} \hat{\Theta}_{M}^{2} U_{\mathrm{PMNS}}^{\dagger},
$$

where $U_{\text {PMNS }}$ is the Pontecorvo-Maki-Nakagawa-Sakata (PMNS) matrix [70,71] with three mixing angles $\theta_{12}$, $\theta_{23}, \theta_{13}$, and a Dirac $C P$ phase $\delta_{\mathrm{CP}}$, while diagonal phase matrix $\hat{\Theta}_{M}$ contains Majorana phases. The overall phase of $\hat{\Theta}_{M}$ is unphysical, and we adopt the convention such that $\hat{\Theta}_{M}=\operatorname{diag}\left(1, e^{i \varphi_{M_{2}}}, e^{i \varphi_{M_{3}}}\right)$. Furthermore, $\hat{m}_{\nu}$ is a real diagonal matrix containing mass eigenvalues of left-handed neutrinos. In Table I, we summarize the parameters in $\hat{m}_{\nu}$ and $U_{\text {PMNS }}$ used in our numerical analysis. Here, we consider both NO and IO cases for the left-handed neutrino masses, taking the lightest neutrino mass of $m_{\nu_{1}}^{2}=1.00 \times$ $10^{-6} \mathrm{eV}^{2}$ (NO case) or $m_{\nu_{3}}^{2}=1.00 \times 10^{-6} \mathrm{eV}^{2}$ (IO case).

Comparing Eqs. (2.19) and (2.20), $\underline{y}_{\nu}$ can be expressed in the following form, i.e., the so-called Casas-Ibarra parametrization $[39]^{5}$ :

$$
\underline{y}_{\nu}=\frac{1}{v_{u}} \underline{\hat{M}}_{N}^{\frac{1}{2}} R \hat{m}_{\nu}^{\frac{1}{2}} \hat{\Theta}_{M} U_{\mathrm{PMNS}}^{\dagger},
$$

where $R$ is an arbitrary complex and orthogonal matrix:

$$
R R^{T}=\mathbf{1}
$$

The two parametrizations of the neutrino Yukawa matrices, Eqs. (2.17) and (2.21), are equivalent. The number of parameters in both parametrizations are summarized in Tables II and III.

The complex orthogonal matrix $R$ can be decomposed into the product of a real orthogonal matrix $O$ and a Hermitian and orthogonal matrix $H$ as $R=O H$. Let us define

$$
n(\theta, \phi) \equiv(\sin \theta \cos \phi, \sin \theta \sin \phi, \cos \theta)
$$

and

\footnotetext{
${ }^{5}$ Square root of a diagonal matrix is understood to be applied to each diagonal element.
} 
TABLE II. The number of real degrees of freedom in the neutrino Yukawa matrix in the parametrization of Eq. (2.17).

\begin{tabular}{ccccc}
\hline \hline$\hat{\Theta}_{l}$ & $\tilde{W}$ & $U$ & $\hat{f}_{\nu}$ & Total \\
\hline 2 & 9 & 4 & 3 & 18 \\
\hline \hline
\end{tabular}

TABLE III. The number of real degrees of freedom in the neutrino Yukawa matrix in CI parametrization, where $\varphi_{\tilde{W}}$ is the overall phase of the matrix $\tilde{W}$.

\begin{tabular}{cccccccc}
\hline \hline$\varphi_{\tilde{W}}$ & $\hat{\Theta}_{l}$ & $\hat{\Theta}_{M}$ & $U_{\mathrm{PMNS}}$ & $\hat{m}_{\nu}$ & $O$ & $H$ & Total \\
\hline 1 & 2 & 2 & 4 & 3 & 3 & 3 & 18 \\
\hline \hline
\end{tabular}

$$
(A(n))_{i j} \equiv \epsilon_{i j k} n_{k}
$$

Then, $H$ can be expressed as

$H(r, n)=e^{i r A(n)}=n n^{T}+\cosh r\left(\mathbf{1}-n n^{T}\right)+i \sinh r A(n)$,

and hence is parametrized by three real parameters $(r, \theta, \phi)$. We can derive another useful expression of $H(r, n)$. For this purpose, we introduce a complex vector

$$
\tilde{n} \equiv \frac{1}{\sqrt{2}}\left(n^{\prime}-i n^{\prime \prime}\right)
$$

where $n^{\prime}$ and $n^{\prime \prime}$ are an arbitrary set of two unit vectors such that $\left\langle n, n^{\prime}, n^{\prime \prime}\right\rangle$ forms a right-handed orthonormal basis of $\mathbb{R}^{3}$. One can easily check that $\left\langle n, \tilde{n}, \tilde{n}^{*}\right\rangle$ forms an orthonormal basis of $\mathbb{C}^{3}$. With these vectors, $H(r, n)$ can be expressed as

$$
H(r, n)=e^{r} \tilde{P}(n)+P(n)+e^{-r} \tilde{P}^{*}(n),
$$

where

$$
P(n) \equiv n n^{T}, \quad \tilde{P}(n) \equiv \tilde{n} \tilde{n}^{\dagger}, \quad \tilde{P}^{*}(n) \equiv \tilde{n}^{*} \tilde{n}^{T} .
$$

Here $P, \tilde{P}$, and $\tilde{P}^{*}$ are orthogonal projection matrices onto $\mathbb{C} n, \mathbb{C} \tilde{n}$, and $\mathbb{C} \tilde{n}^{*}$, respectively. Note that $\tilde{P}$ depends only on $n$ and not on the choice of $n^{\prime}$ and $n^{\prime \prime}$. When $r \gg 1$, the first term in the right-hand side of Eq. (2.27) dominates.

Many of the previous analysis of the flavor violations have not paid significant attention to the effect of the CI parameters, taking $R=\mathbf{1}$ (see, however, [32,43,44,46, $47,54,68]$ ). In addition, it has been often assumed that the right-handed neutrino masses are degenerate, i.e., $\underline{M}_{N_{1}}=\underline{M}_{N_{2}}=\underline{M}_{N_{3}}$. With these simplifications, $\underline{y}_{\nu}=$ $\frac{1}{v_{u}}\left(\underline{\hat{M}}_{N} \hat{m}_{\nu}\right)^{\frac{1}{2}} U_{\mathrm{PMNS}}^{\dagger}$. However, as we will see in the following, parameters in $R$ may significantly affect the $C P$ and flavor violating observables.

\section{NUMERICAL ANALYSIS}

Now, let us numerically evaluate the $C P$ and flavor violating observables. Our primary purpose is to study the effects of CI parameters on electron electric dipole moment (EDM) and branching ratios of lepton flavor violating (LFV) processes. Thus, for simplicity, we assume that the soft SUSY breaking parameters satisfy the socalled minimal supergravity (MSUGRA) model boundary conditions; the soft scalar mass-squared parameters at the Planck scale $Q=M_{\mathrm{Pl}}$ are assumed to be universal (and are equal to $m_{0}^{2}$ ), and trilinear scalar couplings (so-called $A$ terms) are proportional to corresponding Yukawa couplings (with the proportionality constant of $a_{0}$ ).

In our analysis, we calculate the MSSM parameters at the mass scale of MSSM superparticles (which we call MSSM scale). Here are remarks about our calculation:

(i) The input $\mathrm{SM}$ parameters related to low energy observables are

$$
g_{a}, \quad \hat{y}_{u}, \quad \hat{y}_{d}, \quad V_{\mathrm{CKM}}, \quad \hat{y}_{e}, \quad \hat{m}_{\nu}, \quad U_{\mathrm{PMNS}},
$$

where $g_{a}$ (with $a=1-3$ ) are gauge coupling constants, while $V_{\mathrm{CKM}}$ is the Cabibbo-Kobayashi-Maskawa (CKM) matrix. For the boundary conditions of SM couplings at the top mass scale $M_{t}$, we follow [72]. The parameters in the CKM matrix are taken from [1] and set at $M_{t}$; the lightest neutrino mass, which cannot be determined from current neutrino oscillation experiments, is set to be $1 \times 10^{-3} \mathrm{eV}$. For the left-handed neutrino mass eigenstates and the PMNS matrix, we use the values given in Table I at the scale of right-handed neutrino masses, neglecting the renormalization group running of the neutrino mass and mixing parameters below the mass scale of the right-handed neutrinos. At $M_{t}$, two-loop and three-loop SM thresholds are included.

(ii) In addition, we fix other input parameters:

$$
\begin{aligned}
& m_{0}, \quad a_{0}, \quad M_{1 / 2}, \quad \tan \beta, \quad \operatorname{sgn}(\mu), \quad \underline{\hat{M}}_{N}, \\
& \hat{\Theta}_{l}, \quad \hat{\Theta}_{M}, \quad O, \quad H,
\end{aligned}
$$

where $M_{1 / 2}$ is the SU(5) gaugino mass at the Planck scale, $\tan \beta$ is the ratio of the VEVs of up- and downtype Higgs bosons, and $\mu$ is supersymmetric Higgs mass parameter. (Here, we assume that $\mu$, as well as $a_{0}$ and $M_{1 / 2}$, are real.) In the following, we use the fact that, in the decoupling limit of the heavy Higgs doublet, the SM-like Higgs boson, $H_{\mathrm{SM}}$, is given as $H_{\mathrm{SM}}=H_{u} \sin \beta+i \sigma_{2} H_{d}^{\dagger} \cos \beta$ at $M_{S}$. For simplicity, we take $M_{1 / 2}=m_{0}, a_{0}=0$ and $\operatorname{sgn}(\mu)=+$.

(iii) The SM parameters at the MSSM scale is obtained by using the SM renormalization group equations 
(RGEs). In our analysis, the MSSM scale is define as the geometric mean of the stop mass eigenvalues, $M_{S}=\sqrt{m_{\tilde{t}_{1}} m_{\tilde{t}_{2}}}$; in our numerical calculation, $M_{\mathrm{S}}$ is determined iteratively (see the following arguments). At $M_{\mathrm{S}}$, SUSY threshold corrections to the Higgs quartic coupling constant $\lambda$, gauge couplings, and the top Yukawa coupling are included [73].

(iv) The MSSM parameters at the mass scale of the right-handed neutrino masses are obtained by using SOFTSUSY package [74]. The couplings in the MSSM and those of the MSSMNR are matched at the tree level. Notice that each right-handed neutrino decouples from the effective theory at $M_{N_{i}}$, and we use the effective theory without $\bar{N}_{i}$ for the scale $Q<\underline{M}_{N_{i}}$; here, $\bar{N}_{i}$ is defined in the basis in which it becomes the mass eigenstate of $M_{N}\left(Q=\underline{M}_{N_{i}}\right)$.

(v) In order to study the running between the mass scale of the right-handed neutrinos and the GUT scale, we modify SOFTSUSY package with including the coupling constants related to right-handed neutrinos. The RGEs of MSSMNR can be obtained in [49].

(vi) The Yukawa matrices of the MSSMNR and those of the SU(5)NR are matched by using Eqs. (2.14)(2.18) at the GUT scale; in our numerical calculation, we take $M_{\mathrm{GUT}}=2 \times 10^{16} \mathrm{GeV}$. Other parameters are also matched accordingly. At the GUT scale, threshold corrections on down-type and chargedlepton type Yukawa matrices of MSSMNR are imposed following [69] while tree-level matching conditions are adopted for other dimensionless couplings and soft masses.

(vii) In order to take into account the running above the GUT scale, we also implement the RGEs in the SU(5)NR. Here, we assume that the particle content of the SU(5)NR is minimal; $\Phi_{i}, \Psi_{i}, \Upsilon, H, \bar{H}$, and $\Sigma$, as well as the gauge multiplet. The RGEs of the $\mathrm{SU}(5)$ gauge coupling constant and the gaugino mass are obtained based on this particle content. In addition, for simplicity, we assume that the interactions of $\Sigma$ are so weak that their effects on the running are negligible.

(viii) The SUSY breaking parameters at the Planck scale are set by using the parameters $m_{0}, a_{0}$, and $M_{1 / 2}$.

For RGEs, we use two loop (SM and MSSM) and one loop [MSSMNR and SU(5)NR]. However, for the calculation of the Higgs quartic coupling constant, we follow [73] and solve three-loop RGEs between $M_{t}$ and $M_{\mathrm{S}}$.

With the modified SOFTSUSY package explained above, we calculate the MSSM parameters at $Q=M_{\mathrm{S}}$ as follows. For the consistency of boundary conditions at $M_{t}$ and at $M_{\mathrm{Pl}}$, we iterate on $M_{S}$ and $M_{H}$.

(1) We first fix the set of input parameters given in Eq. (3.2). Then, we adopt the temporary values $M_{S}=m_{0}$ and $M_{H}=126 \mathrm{GeV}$.
(2) The boundary conditions for SM couplings at $Q=M_{t}$ are determined with following the procedure given in [72], using $M_{t}$, Higgs mass $M_{H}$, $W$-boson mass $M_{W}, Z$-boson mass $M_{Z}$, the strong coupling constant $\alpha_{s}\left(M_{Z}\right)$ and fine structure constant $\alpha\left(M_{Z}\right)$ as input parameters.

(3) We solve the RGE runnings of the Yukawa coupling constants and the gauge coupling constants from $M_{t}$ to $M_{\mathrm{Pl}}$ and the Planck-scale values of those parameters are determined.

(4) We set the boundary conditions for soft SUSY breaking parameters at $M_{\mathrm{Pl}}$ and run them down to $M_{\mathrm{S}}$.

(5) At $M_{S}$, the SM Higgs quartic coupling $\lambda$ is calculated with including the SUSY threshold corrections. The $\mu$ parameter and $B$ parameter are determined from the condition of successful electroweak symmetry breaking; we require that a linear combination of $H_{u}$ and $H_{d}$, i.e., $H_{u} \sin \beta+i \sigma_{2} H_{d}^{\dagger} \cos \beta$, behaves as the SM Higgs boson, with the condition that it becomes an eigenstate of the Higgs mass-squared matrix with the correct eigenvalue inferred from the $\mathrm{SM}$. Then, in particular, the $\mu$ parameter is given by

$$
\mu^{2}=m_{H_{\mathrm{SM}}}^{2}-\frac{m_{H_{u}}^{2} \tan ^{2} \beta-m_{H_{d}}^{2}}{\tan ^{2} \beta-1},
$$

where $m_{H_{u}}^{2}$ and $m_{H_{d}}^{2}$ are the MSSM Higgs soft mass parameters, and $m_{H_{\mathrm{SM}}}^{2}$ is the Higgs mass squared parameter of the SM; notice that the above relation holds at $Q=M_{S}{ }^{6}$

(6) We run SM couplings from $M_{S}$ to $M_{t}$.

(7) Renew $M_{S}$ from stop masses and $M_{H}$ from SM couplings.

(8) Iterate the steps from 2 to 6 until $M_{S}$ and $M_{H}$ converge.

Once the MSSM parameters at $Q=M_{\mathrm{S}}$ are fixed, $C P$ and LFV observables are calculated by using those parameters. After electroweak symmetry breaking, relevant operators of our interest are given by

$$
\begin{gathered}
\mathcal{L}^{\mathrm{eEDM}}=-\frac{i d_{e}}{2} \bar{\psi}_{e} \sigma^{\mu \nu} \gamma^{5} \psi_{e} F_{\mu \nu}, \\
\mathcal{L}^{\mu \rightarrow e \gamma}=-\frac{1}{2} \bar{\psi}_{e} \sigma^{\mu \nu}\left(a_{L} P_{L}+a_{R} P_{R}\right) \psi_{\mu} F_{\mu \nu},
\end{gathered}
$$

\footnotetext{
${ }^{6}$ We use the tree-level MSSM Higgs mass-squared matrix to calculate $\mu$. The threshold corrections to the MSSM Higgs soft mass parameters affect $\mu$. The effect of the threshold correction on $\mu$ is expected to be of $O(1) \%$ because it is loop suppressed. (Notice that no large logarithmic effect is expected because such effects, particularly from the top Yukawa coupling constant, are taken into account by the renormalization group running below $Q=M_{S}$.) Such an error in the estimation of $\mu$ does not alter our main conclusion on the $C P$ and flavor violating processes given in the next section.
} 
where $F_{\mu \nu}$ is the field strength tensor of the photon, $\psi_{\mu}$ and $\psi_{e}$ are field operators of muon and electron, respectively, and $d_{e}, a_{L}$, and $a_{R}$ are coefficients. $d_{e}$ is the electron EDM while the decay rate of $\mu \rightarrow e \gamma$ process is give by

$$
\Gamma(\mu \rightarrow e \gamma)=\frac{m_{\mu}^{3}}{16 \pi}\left(\left|a_{L}\right|^{2}+\left|a_{R}\right|^{2}\right) .
$$

For the LFV decay processes of $\tau$, like $\tau \rightarrow \mu \gamma$ and $\tau \rightarrow e \gamma$, the operator is like that given in Eq. (3.5) with field operators being properly replaced. For the detail about the calculation of the electron EDM and the LFV decay rates, see, for example, [26,59].

Before showing our numerical results, it is instructive to use the leading-log approximation for the understandings of qualitative behaviors. Above $M_{\mathrm{GUT}}, f_{u}$ contributes to the off-diagonal elements of the right-handed selectron mass matrix $m_{\tilde{e}}$ and $f_{\nu}$ contribute to that of the left-handed slepton mass matrix $m_{\tilde{l}}$. Below $M_{\mathrm{GUT}}$, there is no extra violation production in $m_{\tilde{e}}$ from Yukawa interactions but $m_{\tilde{l}}$ still acquires off-diagonal elements from neutrino-type Yukawa interactions. Assuming a universality of the right-handed neutrino masses, the leading-log approximation gives

$$
\begin{aligned}
& \left(m_{\tilde{l}}^{2}\right)_{i j} \simeq m_{0}^{2} \delta_{i j}-\frac{1}{8 \pi^{2}}\left(3 m_{0}^{2}+a_{0}^{2}\right)\left(\hat{\Theta}_{l}^{*} \underline{y}_{\nu}^{\dagger} \underline{-}_{\nu} \hat{\Theta}_{l}\right)_{i j} \log \left(\frac{M_{\mathrm{Pl}}}{M_{N_{R}}}\right), \\
& \left(m_{\tilde{e}}^{2}\right)_{i j} \simeq m_{0}^{2} \delta_{i j}-\frac{3}{8 \pi^{2}}\left(3 m_{0}^{2}+a_{0}^{2}\right)\left(V^{T} \hat{f}_{u}^{2} V^{*}\right)_{i j} \log \left(\frac{M_{\mathrm{Pl}}}{M_{\mathrm{GUT}}}\right),
\end{aligned}
$$

where $M_{N_{R}}$ is the universal right-handed neutrino mass. The off-diagonal elements of $m_{\tilde{l}}^{2}$ are approximately proportional to the corresponding elements of $\hat{\Theta}_{l}^{*} \underline{y}_{\nu}^{\dagger} \underline{y}_{\nu} \hat{\Theta}_{l}$. When the $r$ parameter is sizable we can find

$\hat{\Theta}_{l}^{*} \underline{y}_{\nu}^{\dagger} \underline{y}_{\nu} \hat{\Theta}_{l} \simeq \frac{e^{2 r}}{v_{u}^{2}} \operatorname{tr}\left(\tilde{P}(n) O^{T} \underline{\hat{M}}_{N} O\right) \tilde{U} \hat{m}_{\nu}^{\frac{1}{2}} \tilde{P}(n) \hat{m}_{\nu}^{\frac{1}{2}} \tilde{U}^{\dagger}+O\left(e^{r}\right)$,

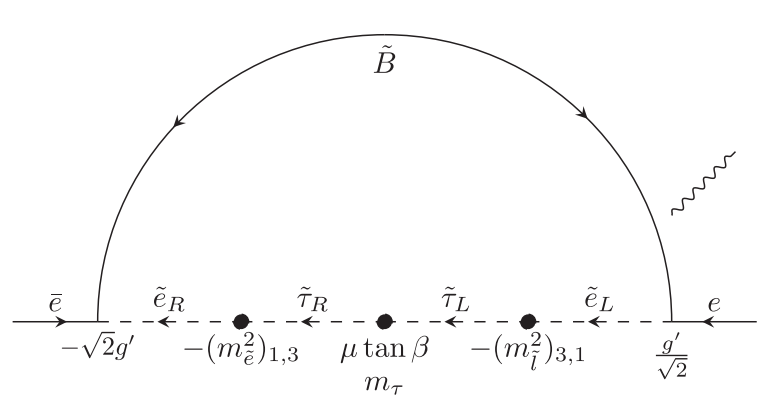

where $\tilde{U} \equiv \hat{\Theta}_{l}^{*} U_{\mathrm{PMNS}} \hat{\Theta}_{M}^{*}$. The CI parameters may enhance the off-diagonal elements of $m_{\tilde{l}}^{2}$ because the magnitude of $\underline{y}_{\nu}^{\dagger} \underline{y}_{\nu}$ is proportional to $e^{2 r}$. From Eqs. (3.7) and (3.9), one can see that the renormalization group effects on $m_{\tilde{l}}^{2}$ is suppressed when the mass scale of the right-handed neutrinos becomes smaller. Thus, when the effects of the CI parameters are neglected, the $C P$ and flavor violations due to the renormalization group effects are highly suppressed when the mass scale of the right-handed neutrinos is much smaller than $\sim 10^{14} \mathrm{GeV}$. With the CI parameters, this may not be the case. One can see that, when the $r$ parameter is larger than $\sim 1$, the renormalization group effects can be sizable even when the right-handed neutrinos are relatively light. In the following, we will see that the enhancement due to the CI parameters can indeed enhance the electron EDM and LFV decay rates.

The off-diagonal elements of the slepton mass matrix become the sources of $C P$ and flavor violations. Although we numerically calculate the electron EDM and LFV decay rates in the mass basis, with which the effects of the offdiagonal elements are taken into account at all orders, it is suggestive to consider the mass insertion method to understand the behaviors of the results. Figure 2 shows the examples of the diagrams contributing to the electron EDM and $\mu \rightarrow e \gamma$ process in the mass insertion approximation. In fact, when $\tan \beta \gg 1$, the dominant contributions to $\mu \rightarrow e \gamma$ originates from a diagram with a mass insertion of $\left(m_{\tilde{l}}\right)_{1,2}$. For the electron EDM, the dominant contribution is from a diagram with mass insertions of $\left(m_{\tilde{e}}\right)_{1,3}$ and $\left(m_{\tilde{l}}\right)_{1,3}$, if there is no $C P$ phase in $\mu$ parameter (see Fig. 2). For the choice of parameters we adopt in the following analysis, we found that the diagrams shown in Fig. 2 become dominant for the electron EDM and the decay rate for the process $\mu \rightarrow e \gamma$.

Now, we show the results of our numerical calculations. Unless otherwise stated, (i) we take $\tan \beta=8$ and $\mathrm{m}_{0}=10 \mathrm{TeV}$, which give $M_{H} \simeq 126 \mathrm{GeV}$, and (ii) we assume the normal ordering for the left-handed neutrino masses. We neglect the effects of Majorana phases and simply set $\hat{\Theta}_{M}=\mathbf{1}$. The three types of structures of $\hat{\underline{M}}_{N}$ and $O$ are adopted:

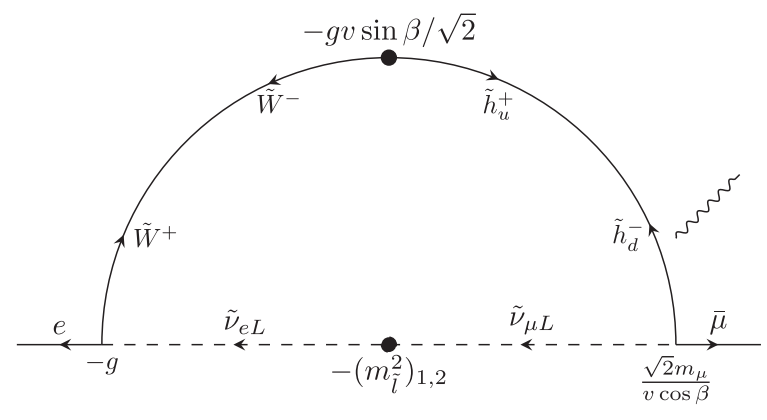

FIG. 2. Examples of the mass insertion contributions to $d_{e}$ (left) and to $\operatorname{Br}(\mu \rightarrow e \gamma)$ (right). 

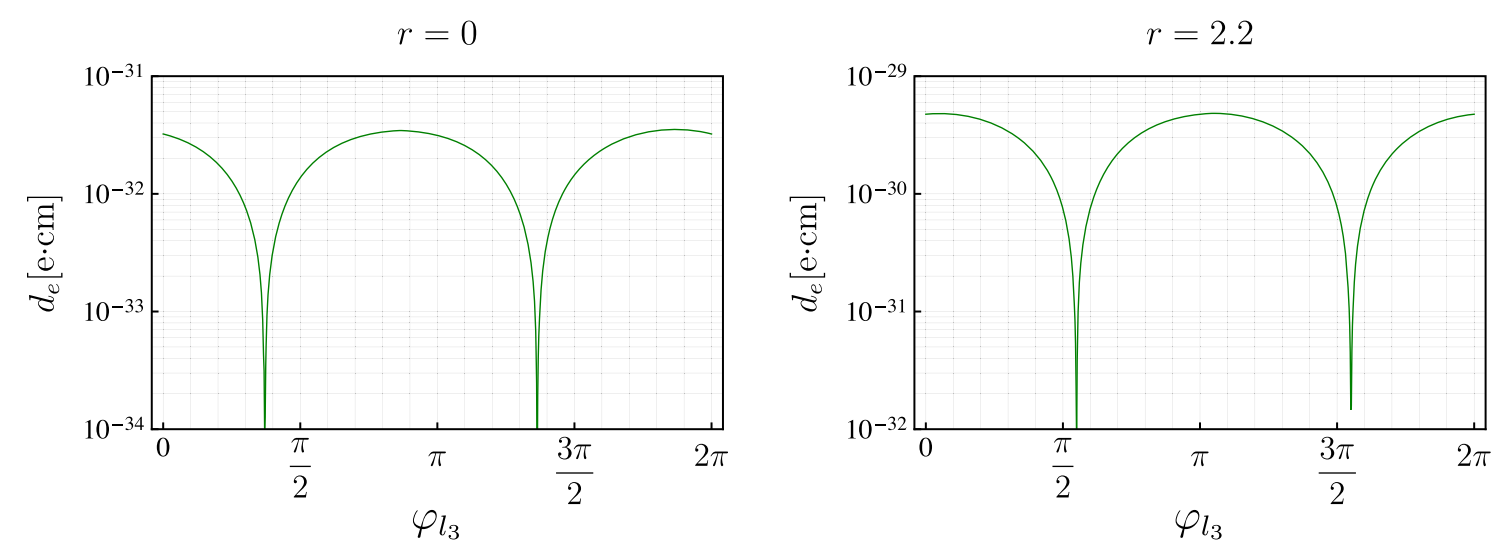

FIG. 3. The dependence of $d_{e}$ on GUT phase $\varphi_{l_{3}}$ with $r=0$ (left) or $r=2.2$ (right). Here $\tan \beta=8, m_{0}=10 \mathrm{TeV}, M_{N_{R}}=10^{13} \mathrm{GeV}$, $(\theta, \phi)=\left(\frac{\pi}{2}, 0\right), \hat{\Theta}_{M}=1$ and $\varphi_{l_{2}}=0$.

(i) (U) Universal:

$$
O^{T} \underline{\hat{M}}_{N} O=M_{N_{R}} \mathbf{1}
$$

(ii) (H) Hierarchical:

$$
O^{T} \underline{\underline{M}}_{N} O=M_{N_{R}}\left(\begin{array}{ccc}
10^{-2} & 0 & 0 \\
0 & 10^{-1} & 0 \\
0 & 0 & 1
\end{array}\right) .
$$

(iii) (IH) Inverse hierarchical:

$$
O^{T} \underline{\hat{M}}_{N} O=M_{N_{R}}\left(\begin{array}{ccc}
1 & 0 & 0 \\
0 & 10^{-1} & 0 \\
0 & 0 & 10^{-2}
\end{array}\right) .
$$

The Yukawa couplings may blow up if the CI parameter $r$ is too large. In such a case, the perturbative calculation becomes unreliable. In order to avoid the blow up of the Yukawa couplings, we impose the following constraints on the neutrino Yukawa couplings at any renormalization scale:

$$
\frac{\operatorname{tr}\left(f_{\nu}^{\dagger} f_{\nu}\right)}{(4 \pi)^{2}}<1, \quad \frac{\operatorname{tr}\left(y_{\nu}^{\dagger} y_{\nu}\right)}{(4 \pi)^{2}}<1
$$

In Fig. 3, we show how the electron EDM depends on the GUT phases $\varphi_{l_{3}}$, adopting the structure (U) of right-handed neutrino masses and $M_{N_{R}}=10^{13} \mathrm{GeV}$. Here, we take $R=\mathbf{1}$ (left) and $(r, \theta, \phi)=(2.2, \pi / 2,0)$ (right). As shown in Fig. 3, the electron EDM is sensitive to $\varphi_{l_{3}}$. This is because, through the renormalization group effects, $\varphi_{l_{3}}$ affects the complex phase of $\left(m_{\tilde{l}}\right)_{31}$, which the electron EDM is (approximately) proportional to. We can see that the position of the peak is shifted with the introduction of the CI parameters because they contain $C P$ phases. In the following analysis, in order to (approximately) maximize the electron EDM, we tune the GUT phase $\varphi_{l_{3}}$ so that the contribution of the mass insertion diagram shown in Fig. 2 (left), which gives the dominant contribution to the electron EDM in most of the parameter region in our study, is maximized. ${ }^{7}$ We have also studied how the electron EDM depends on the phase $\varphi_{l_{2}}$, and confirmed that such a dependence is weak.

Figure 4 shows the contours of constant Higgs mass, maximized electron EDM and $\operatorname{Br}(\mu \rightarrow e \gamma)$ on $\left(m_{0}, \tan \beta\right)$ plane, taking $M_{N_{R}}=10^{13} \mathrm{GeV}$. In the figure, we also show the contours on which $d_{e}$ and $\operatorname{Br}(\mu \rightarrow e \gamma)$ become equal to the current experimental upper bounds; the upper bound on $d_{e}$ is given by ACME as [75]

$$
d_{e}<1.1 \times 10^{-29} e \mathrm{~cm},
$$

while the upper bound on the branching ratio for $\mu \rightarrow e \gamma$ process is given by the MEG experiment as [76]

$$
\operatorname{Br}(\mu \rightarrow e \gamma)<4.2 \times 10^{-13} .
$$

The left plot is for the case of $R=\mathbf{1}$, while the right one is for the case of $(r, \theta, \phi)=(2.2, \pi / 2,0)$. We see that CI parameters have little influence on Higgs mass, but can significantly enhance $d_{e}$ and $\operatorname{Br}(\mu \rightarrow e \gamma)$. This is because, as we increase the $r$ parameter, the Yukawa couplings can become larger [see Eq. (3.9)], which enhances the renormalization group effects on $\left(m_{\tilde{l}}^{2}\right)_{i j}$. Because the electron $\mathrm{EDM}$ and $\operatorname{Br}(\mu \rightarrow e \gamma)$ are sensitive to the off-diagonal elements of slepton mass squared matrices, the proper

\footnotetext{
${ }^{7}$ If $r$ is large enough, then other mass-insertion diagrams with multiple insertions of $\left(m_{\tilde{l}}\right)_{i j}$ become non-negligible. On the other hand, as $\underline{y}_{\nu}$ is small, the diagrams other than the left one of Fig. 2 become sizable.
} 

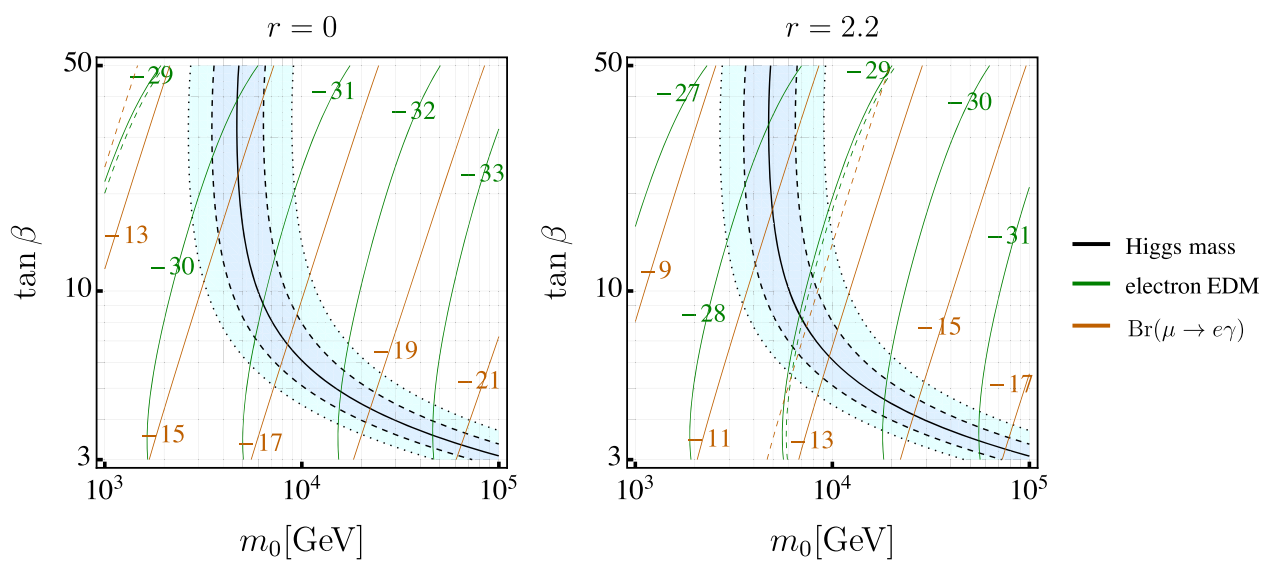

FIG. 4. Higgs mass (black), electron $\operatorname{EDM}$ (green), and $\operatorname{Br}(\mu \rightarrow e \gamma)$ (orange) on $\left(m_{0}\right.$, $\left.\tan \beta\right)$ plane with $r=0$ or $r=2.2$. Here, we take $M_{N_{R}}=10^{13} \mathrm{GeV},(\theta, \phi)=\left(\frac{\pi}{2}, 0\right), \hat{\Theta}_{M}=\mathbf{1}$ and $\varphi_{l_{2}}=0 . \varphi_{l_{3}}$ is chosen to maximize electron EDM. The black lines are for $M_{H}=$ $123,124,125,126,127 \mathrm{GeV}$ from left to right. The numbers in the figure are $\log _{10} d_{e}$ (green) and $\log _{10} \operatorname{Br}(\mu \rightarrow e \gamma)$ (orange). The green and orange dashed lines are contours on which $d_{e}$ and $\operatorname{Br}(\mu \rightarrow e \gamma)$ become equal to the current experimental upper bounds, Eqs. (3.14) and (3.15), respectively.

introduction of the CI parameters has significant impact on the $C P$ and flavor violating observables.

In Fig. 5, we show contours of constant maximized electron $\mathrm{EDM}$ and $\operatorname{Br}(\mu \rightarrow e \gamma)$ on $\left(M_{N_{R}}, r\right)$ plane, taking

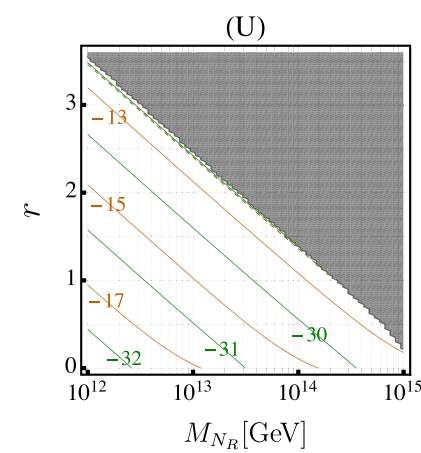

(IH)

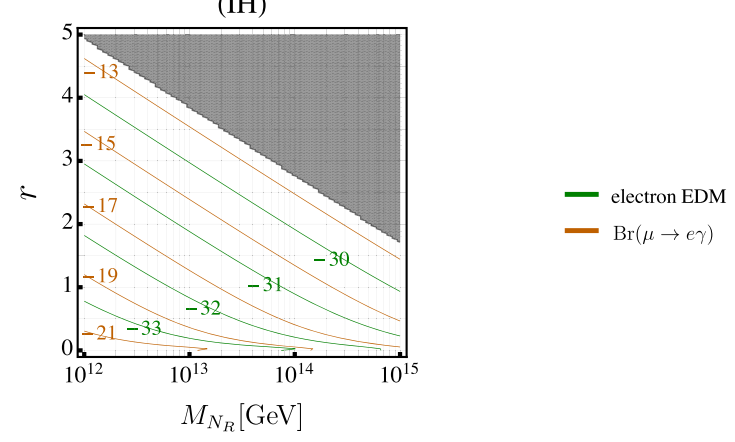

FIG. 5. The electron $\mathrm{EDM}$ (green) and $\operatorname{Br}(\mu \rightarrow e \gamma)$ (orange) on $\left(M_{N_{R}}, r\right)$ plane for the structures $(\mathrm{U}),(\mathrm{H})$, and $(\mathrm{IH})$ of $O^{T} \underline{\hat{M}}_{N} O$. Here we assume the normal ordering of left-handed neutrinos masses. The numbers in the figure are $\log _{10} d_{e}$ (green) and $\log _{10} \operatorname{Br}(\mu \rightarrow e \gamma)$ (orange). We take $\tan \beta=8, m_{0}=10 \mathrm{TeV}$, $(\theta, \phi)=(\pi / 2,0), \hat{\Theta}_{M}=\mathbf{1}$ and $\varphi_{l_{2}}=0 . \varphi_{l_{3}}$ is chosen to maximize $d_{e}$. $(\theta, \phi)=(\pi / 2,0)$, assuming the normal ordering of the lefthanded neutrino masses. In the figure, we shade the regions in which the perturbativity of the Yukawa couplings breaks down. Over the wide range of the parameter space, we have checked that the mass-insertion diagrams shown in Fig. 2 are dominant. We can see that the maximal possible values of the electron EDM and $\operatorname{Br}(\mu \rightarrow e \gamma)$ are insensitive to the scale and the structure of the right-handed neutrino mass matrix. This is because the enhancement of the neutrino Yukawa couplings due to the factor of $e^{2 r}$ compensates the suppression due to the smallness of the right-handed neutrino mass [see Eq. (3.9)]. Figure 6 shows the contours of constant $\operatorname{Br}(\tau \rightarrow e \gamma)$ and $\operatorname{Br}(\tau \rightarrow \mu \gamma)$. They are also enhanced by CI parameters but are fairly below the current experimental bounds. In Fig. 7, we also show how $d_{e}$ and

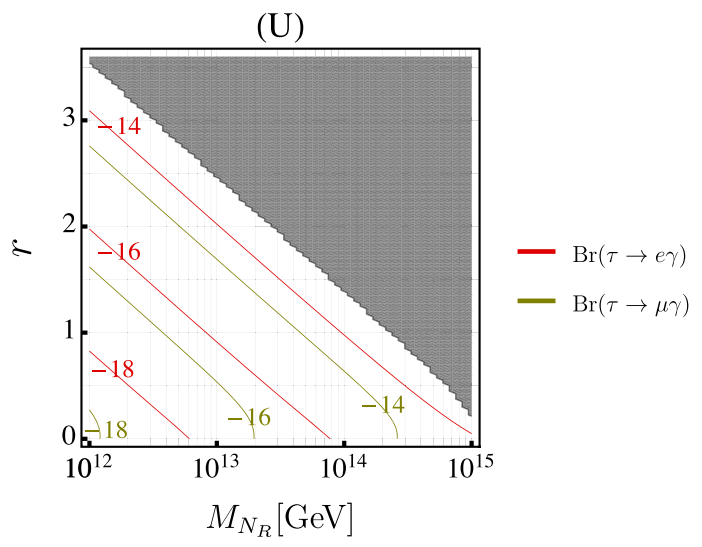

FIG. 6. $\operatorname{Br}(\tau \rightarrow e \gamma)$ (red) and $\operatorname{Br}(\tau \rightarrow \mu \gamma)$ (yellow) on $\left(M_{N_{R}}, r\right)$ plane for the universal-type right-handed neutrino masses. Here we assume normal ordering of left-handed neutrino masses. The numbers are $\log _{10} \operatorname{Br}(\tau \rightarrow e \gamma)$ (red) and $\log _{10} \operatorname{Br}(\tau \rightarrow \mu \gamma$ ) (yellow). Other parameters are the same as those in Fig. 5. 

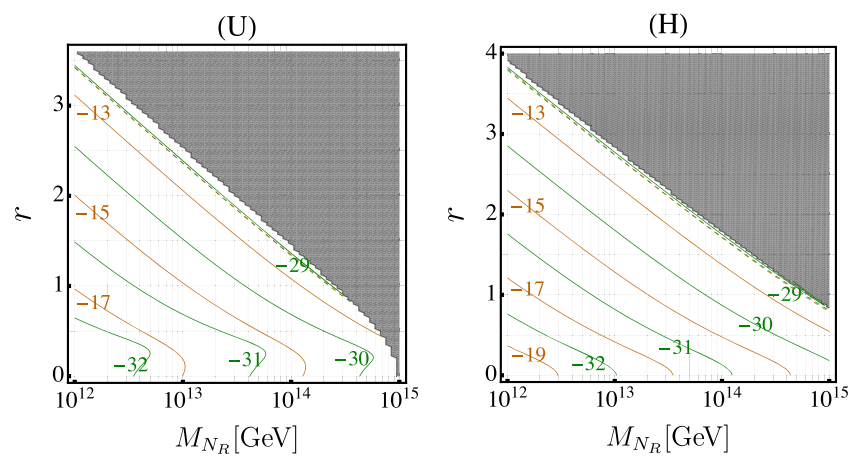

(IH)

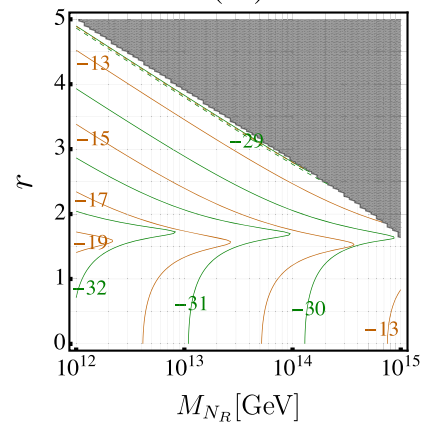

- electron EDM

- $\operatorname{Br}(\mu \rightarrow e \gamma)$

FIG. 7. The electron $\mathrm{EDM}$ (green) and $\operatorname{Br}(\mu \rightarrow e \gamma)$ (orange) on $\left(M_{N_{R}}, r\right)$ plane for the structures $(\mathrm{U}),(\mathrm{H})$, and (IH) of $O^{T} \underline{\hat{M}}_{N} O$. Here we assume the inverted ordering of left-handed neutrinos masses. The numbers in the figure are $\log _{10} d_{e}$ (green) and $\log _{10} \operatorname{Br}(\mu \rightarrow e \gamma)$ (orange). Other parameters are the same as those in Fig. 5.

$\operatorname{Br}(\mu \rightarrow e \gamma)$ behave on a $\left(M_{N_{R}}, r\right)$ plane, assuming the inverted ordering of the left-handed neutrino masses; again, the maximal possible values of the electron EDM and $\operatorname{Br}(\mu \rightarrow e \gamma)$ do not have significant dependence on the mass scale of the right-handed neutrinos.

Figures 5-7 show our main conclusion that the leptonic $C P$ and flavor violating signals through the renormalization group effects can be sizable irrespective of the mass scale of right-handed neutrinos. This is a contrast to the case without taking into account the effects of the CI parameters; without the CI parameters, the neutrino Yukawa coupling constants become tiny when the righthanded neutrinos are much lighter than $\sim 10^{14} \mathrm{GeV}$. In other words, we have a chance to observe the leptonic $C P$ and/or flavor violating signals from the renormalization group effect even when the right-handed neutrino masses are relatively small.

In Fig. 8, we show how large the $r$ parameter can be on $(\theta, \phi)$ plane, taking $(\mathrm{U})$ universal right-handed neutrinos with $M_{N_{R}}=10^{13} \mathrm{GeV}$. With the choice of parameters adopted in Fig. 8, $r$ is required to be smaller than about

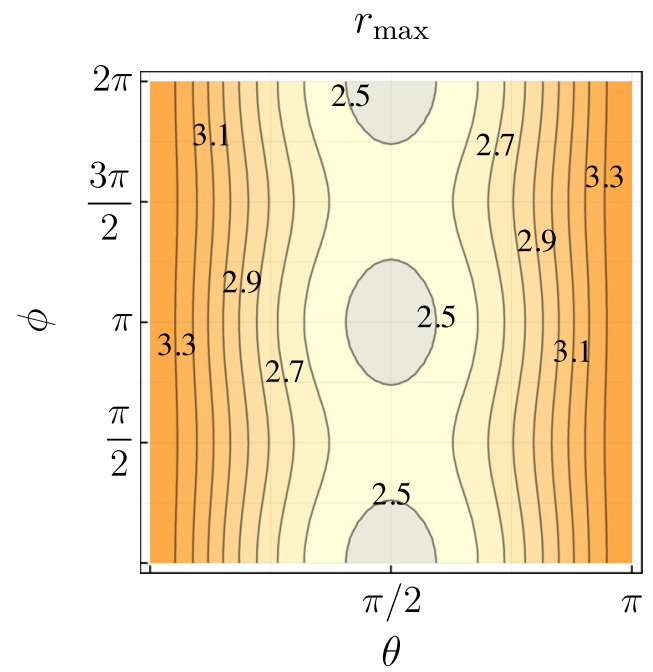

FIG. 8. The maximized $r$ parameter under the perturbativity constraints; the numbers in the figure show the value of $r$. Here, we take the universal-type right-handed neutrino mass parameters with $M_{N_{R}}=10^{13} \mathrm{GeV} \cdot \tan \beta=8, m_{0}=10 \mathrm{TeV}, \hat{\Theta}_{M}=\mathbf{1}$, and $\varphi_{l_{2}}=0 . \varphi_{l_{3}}$ is chosen to maximize electron EDM.

2.5-3.3. Using the maximal possible value of $r$ given in Fig. 8, we calculate the $C P$ and flavor violating observables. In Fig. 9, we show maximized electron $\mathrm{EDM}, \operatorname{Br}(\mu \rightarrow e \gamma), \operatorname{Br}(\tau \rightarrow e \gamma)$, and $\operatorname{Br}(\tau \rightarrow e \gamma)$. We can see that some of the observables are suppressed at particular points on the $(\theta, \phi)$ plane and that the points of the suppressions are correlated for different observables. These are because, at the points of the suppressions, two of $\left(m_{\tilde{l}}\right)_{i j}$ (with $i \neq j$ ) are simultaneously suppressed while the others are sizable. This can be understood as follows. When $r$ is large, the off-diagonal elements of $m_{\tilde{l}}$ can be approximated as

$$
\left(m_{\tilde{l}}\right)_{i j} \propto u_{i} u_{j}^{*},
$$

where [see Eq. (3.9)]

$$
u\left(\theta, \phi, \varphi_{M_{k}}, \varphi_{l_{k}}\right) \equiv \hat{\Theta}_{l}^{*} U_{\mathrm{PMNS}} \hat{\Theta}_{M}^{*} \hat{m}_{\nu}^{\frac{1}{2}} \tilde{n} .
$$

Thus, if one of the elements $u_{i}$ becomes accidentally small, $\left(m_{\tilde{l}}\right)_{i j}(j=1-3)$ are all suppressed, resulting in the correlation of the suppression points shown in Fig. 9. For example, if $u_{1}$ is close to $0,\left(m_{\tilde{l}}\right)_{1,2}$ and $\left(m_{\tilde{l}}\right)_{1,3}$, and hence $d_{e}, \operatorname{Br}(\mu \rightarrow e \gamma)$ and $\operatorname{Br}(\tau \rightarrow e \gamma)$, become simultaneously suppressed; for the present choice of parameters, this happens when $(\theta, \phi) \simeq(0.42 \pi, 1.18 \pi)$ and $(0.58 \pi, 0.52 \pi)$. 

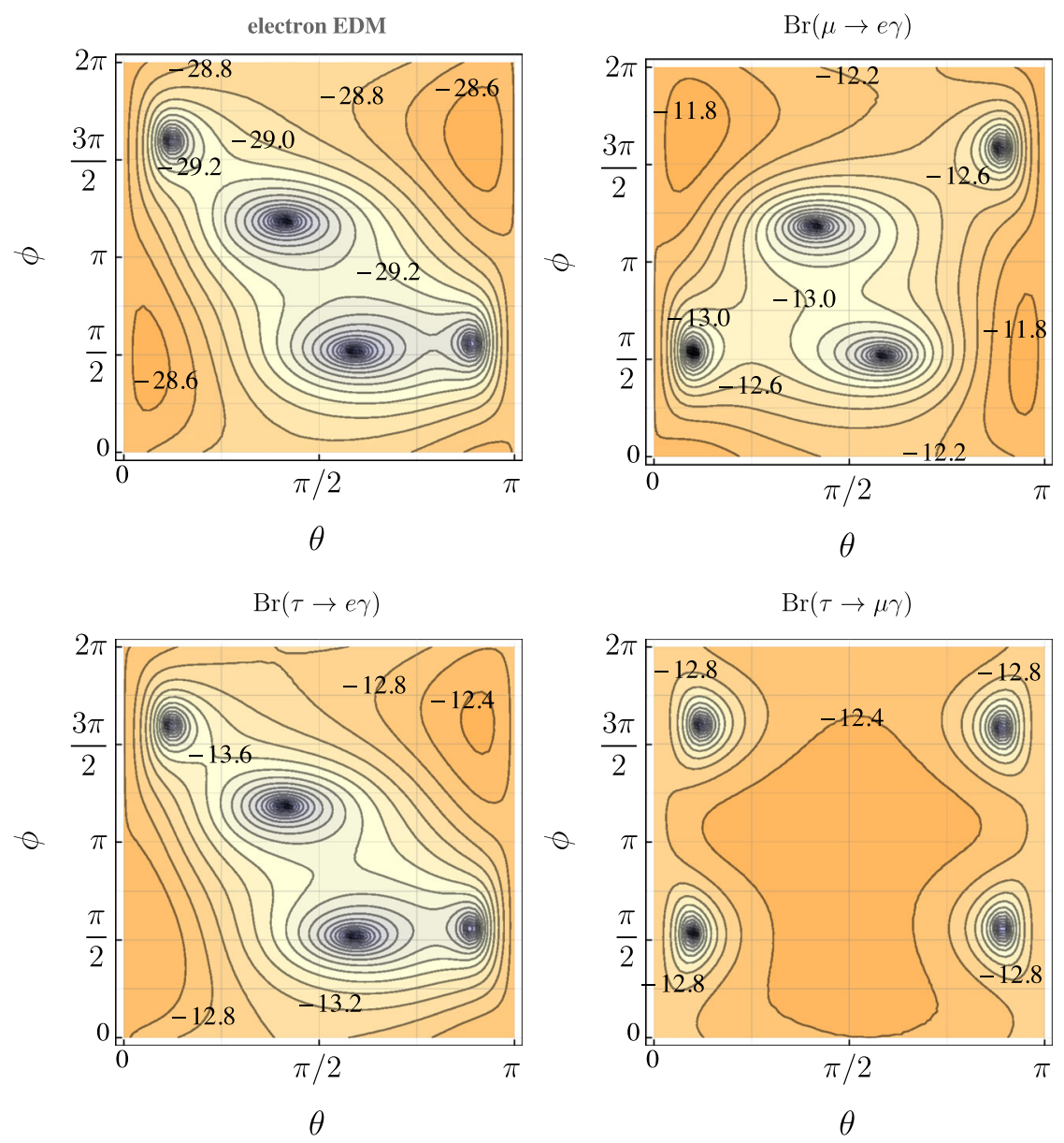

FIG. 9. Contours of constant electron EDM (upper left), $\operatorname{Br}(\mu \rightarrow e \gamma)$ (upper right), and $\operatorname{Br}\left(\tau \rightarrow l_{j} \gamma\right)$ (lower), calculated with the maximal possible value of $r$ shown in Fig. 8. The numbers in the figures are $\log _{10} d_{e}$ (upper $\operatorname{left}_{\text {) }} \log _{10} \operatorname{Br}(\mu \rightarrow e \gamma)$ (upper right), and $\log _{10} \operatorname{Br}\left(\tau \rightarrow l_{j} \gamma\right)$ (lower). The universal-type right-handed neutrino mass parameters are taken with $M_{N_{R}}=10^{13} \mathrm{GeV} . \tan \beta=8$, $m_{0}=10 \mathrm{TeV}, \hat{\Theta}_{M}=\mathbf{1}$, and $\varphi_{l_{2}}=0 . \varphi_{l_{3}}$ is chosen to maximize electron EDM.

\section{CONCLUSIONS AND DISCUSSION}

We have studied the leptonic $C P$ and flavor violating observables, i.e., the electron EDM $d_{e}$ and the branching rations of lepton flavor violating decays $\operatorname{Br}\left(l_{i} \rightarrow l_{j} \gamma\right)$, in the minimal supersymmetric SU(5) GUT with three righthanded neutrinos. We paid particular attention to the effects of the CI parameters $R=O H(r, \theta, \phi)$ in the neutrino Yukawa matrix, which has not been studied extensively before. With the assumption of the universality boundary conditions for soft SUSY breaking masses, we have calculated the electron $\mathrm{EDM}$ and $\operatorname{Br}\left(l_{i} \rightarrow l_{j} \gamma\right)$ with varying CI parameters as well as the MSSM and GUT parameters. Imposing Higgs mass constraints as well as other constraints from low-energy observations, we have studied how the $C P$ and flavor violating observables behave.

In SUSY models, the off-diagonal elements of the slepton mass matrices are induced by renormalization group effects in particular when there exist right-handed neutrinos with sizable neutrino Yukawa couplings or when quarks and leptons are unified into same multiplets of GUT. The off-diagonal elements of the slepton mass matrices become sources of the leptonic $C P$ and flavor violating observables, i.e., the electron $\mathrm{EDM}$ and $\operatorname{Br}\left(l_{i} \rightarrow l_{j} \gamma\right)$. Without taking into account the effects of the CI parameters, effects of the right-handed neutrinos on the renormalization group runnings become irrelevant if the mass scale of the right-handed neutrinos is small; this is because, assuming the seesaw formula for the active neutrino masses, the neutrino Yukawa coupling is suppressed as the right-handed neutrino becomes lighter. Effects of the CI parameters may compensate such an effect, and we found that the maximal possible values of $d_{e}$ and $\operatorname{Br}\left(l_{i} \rightarrow l_{j} \gamma\right)$ are insensitive to the structure of right-handed neutrino masses $\underline{\hat{M}}_{N}$ and the orthogonal matrix $O$. Especially, there are points where two of three independent off-diagonal elements are simultaneously suppressed. Therefore, experimental studies of all the $C P$ and flavor violating observables are important to probe the model. 
One interesting implication of our analysis should be on the leptogenesis scenario [16], in which the lepton asymmetry generated by the decay of the right-handed neutrino is converted to the baryon asymmetry of the Universe. In a simple leptogenesis scenario, the mass scale of the lightest right-handed neutrino is required to be larger than $\sim 10^{9-10} \mathrm{GeV}[77,78]$, while it should be smaller than the reheating temperature after inflation in order not to dilute the generated baryon asymmetry. The total amount of the baryon asymmetry generated by the leptogenesis scenario depends on the detailed structure of the neutrino Yukawa couplings and neutrino mass matrix. The detailed analysis of the leptonic $C P$ and flavor violating observables in connection with the leptogenesis scenario is left for a future work [79].

In this paper, we have concentrated on leptonic $C P$ and flavor violations. In SUSY GUT with right-handed neutrinos, however, it is also notable that sizable offdiagonal elements of squark mass matrices may be also generated via the renormalization group effects. In particular, above the GUT scale, the neutrino Yukawa interactions affect the renormalization group runnings of the right-handed sdown mass matrix; such an effect should also be sensitive to the CI parameters. The renormalization group effects on the squark mass matrices, as well as hadronic $C P$ and flavor violations in connection with such effects, will be studied elsewhere [79].

\section{ACKNOWLEDGMENTS}

The work of K. H. is partially supported by the Program for Leading Graduate Schools, Excellence in Photon Science, Japan. The work of T. M. is supported by JSPS KAKENHI Grants No. 16H06490 and No. 18K03608.
[1] P. A. Zyla et al. (Particle Data Group), Review of particle physics, Prog. Theor. Exp. Phys. (2020), 083C01.

[2] Y. Okada, M. Yamaguchi, and T. Yanagida, Upper bound of the lightest Higgs boson mass in the minimal supersymmetric standard model, Prog. Theor. Phys. 85, 1 (1991).

[3] Y. Okada, M. Yamaguchi, and T. Yanagida, Renormalization group analysis on the Higgs mass in the softly broken supersymmetric standard model, Phys. Lett. B 262, 54 (1991).

[4] J. R. Ellis, G. Ridolfi, and F. Zwirner, Radiative corrections to the masses of supersymmetric Higgs bosons, Phys. Lett. B 257, 83 (1991).

[5] H. E. Haber and R. Hempfling, Can the Mass of the Lightest Higgs Boson of the Minimal Supersymmetric Model Be Larger Than $\mathrm{m}(\mathrm{Z})$ ? Phys. Rev. Lett. 66, 1815 (1991).

[6] J. D. Wells, PeV-scale supersymmetry, Phys. Rev. D 71, 015013 (2005).

[7] G. F. Giudice, M. A. Luty, H. Murayama, and R. Rattazzi, Gaugino mass without singlets, J. High Energy Phys. 12 (1998) 027.

[8] M. Ibe, T. Moroi, and T. T. Yanagida, Possible signals of Wino LSP at the large hadron collider, Phys. Lett. B 644, 355 (2007).

[9] M. Ibe and T. T. Yanagida, The lightest Higgs boson mass in pure gravity mediation model, Phys. Lett. B 709, 374 (2012).

[10] N. Arkani-Hamed, A. Gupta, D. E. Kaplan, N. Weiner, and T. Zorawski, Simply unnatural supersymmetry, arXiv: 1212.6971.

[11] N. Sakai and T. Yanagida, Proton decay in a class of supersymmetric grand unified models, Nucl. Phys. B197, 533 (1982).

[12] S. Weinberg, Supersymmetry at ordinary energies. 1. Masses and conservation laws, Phys. Rev. D 26, 287 (1982).
[13] P. Minkowski, $\mu \rightarrow e \gamma$ at a rate of one out of $10^{9}$ muon decays? Phys. Lett. 67B, 421 (1977).

[14] T. Yanagida, Horizontal gauge symmetry and masses of neutrinos, Conf. Proc. C 7902131, 95 (1979).

[15] M. Gell-Mann, P. Ramond, and R. Slansky, Complex spinors and unified theories, Conf. Proc. C 790927, 315 (1979).

[16] M. Fukugita and T. Yanagida, Baryogenesis without grand unification, Phys. Lett. B 174, 45 (1986).

[17] L. J. Hall, V. A. Kostelecky, and S. Raby, New Flavor Violations in Supergravity Models, Nucl. Phys. B267, 415 (1986).

[18] F. Borzumati and A. Masiero, Large Muon and Electron Number Violations in Supergravity Theories, Phys. Rev. Lett. 57, 961 (1986).

[19] R. Barbieri and L. J. Hall, Signals for supersymmetric unification, Phys. Lett. B 338, 212 (1994).

[20] S. Dimopoulos and L. J. Hall, Electric dipole moments as a test of supersymmetric unification, Phys. Lett. B 344, 185 (1995).

[21] R. Barbieri, L. J. Hall, and A. Strumia, Violations of lepton flavor and $C P$ in supersymmetric unified theories, Nucl. Phys. B445, 219 (1995).

[22] J. Hisano, T. Moroi, K. Tobe, M. Yamaguchi, and T. Yanagida, Lepton flavor violation in the supersymmetric standard model with seesaw induced neutrino masses, Phys. Lett. B 357, 579 (1995).

[23] S. Dimopoulos and D. W. Sutter, The supersymmetric flavor problem, Nucl. Phys. B452, 496 (1995).

[24] P. Ciafaloni, A. Romanino, and A. Strumia, Lepton flavor violations in $\mathrm{SO}(10)$ with large tan beta, Nucl. Phys. B458, 3 (1996).

[25] N. Arkani-Hamed, H. C. Cheng, and L. J. Hall, Flavor mixing signals for realistic supersymmetric unification, Phys. Rev. D 53, 413 (1996). 
[26] J. Hisano, T. Moroi, K. Tobe, and M. Yamaguchi, Lepton flavor violation via right-handed neutrino Yukawa couplings in supersymmetric standard model, Phys. Rev. D 53, 2442 (1996).

[27] R. Barbieri, A. Romanino, and A. Strumia, Electric dipole moments as signals of supersymmetric unification, Phys. Lett. B 369, 283 (1996).

[28] F. Gabbiani, E. Gabrielli, A. Masiero, and L. Silvestrini, A complete analysis of FCNC and $C P$ constraints in general SUSY extensions of the Standard Model, Nucl. Phys. B477, 321 (1996).

[29] J. Hisano, T. Moroi, K. Tobe, and M. Yamaguchi, Exact event rates of lepton flavor violating processes in supersymmetric SU(5) model, Phys. Lett. B 391, 341 (1997); Erratum, Phys. Lett. B 397, 357 (1997).

[30] A. Romanino and A. Strumia, Electric dipole moments from Yukawa phases in supersymmetric theories, Nucl. Phys. B490, 3 (1997).

[31] J. Hisano, M. M. Nojiri, Y. Shimizu, and M. Tanaka, Lepton flavor violation in the left-handed slepton production at future lepton colliders, Phys. Rev. D 60, 055008 (1999).

[32] J. Hisano and D. Nomura, Solar and atmospheric neutrino oscillations and lepton flavor violation in supersymmetric models with the right-handed neutrinos, Phys. Rev. D 59, 116005 (1999).

[33] Y. Okada, K. i. Okumura, and Y. Shimizu, Mu-> e gamma and mu-> 3 e processes with polarized muons and supersymmetric grand unified theories, Phys. Rev. D 61, 094001 (2000).

[34] J. R. Ellis, M. E. Gomez, G. K. Leontaris, S. Lola, and D. V. Nanopoulos, Charged lepton flavor violation in the light of the Super-Kamiokande data, Eur. Phys. J. C 14, 319 (2000).

[35] W. Buchmuller, D. Delepine, and L. T. Handoko, Neutrino mixing and flavor changing processes, Nucl. Phys. B576, 445 (2000).

[36] S. Baek, T. Goto, Y. Okada, and K. i. Okumura, Neutrino oscillation, SUSY GUT and B decay, Phys. Rev. D 63, 051701 (2001).

[37] T. Moroi, Effects of the right-handed neutrinos on Delta $S=2$ and Delta $B=2$ processes in supersymmetric $\mathrm{SU}(5)$ model, J. High Energy Phys. 03 (2000) 019.

[38] T. Moroi, $C P$ violation in $B_{d} \rightarrow \psi K_{s}$ in SUSY GUT with right-handed neutrinos, Phys. Lett. B 493, 366 (2000).

[39] J.A. Casas and A. Ibarra, Oscillating neutrinos and $\mu \rightarrow e, \gamma$, Nucl. Phys. B618, 171 (2001).

[40] S. Baek, T. Goto, Y. Okada, and K. i. Okumura, Muon anomalous magnetic moment, lepton flavor violation, and flavor changing neutral current processes in SUSY GUT with right-handed neutrino, Phys. Rev. D 64, 095001 (2001).

[41] N. Akama, Y. Kiyo, S. Komine, and T. Moroi, CP violation in kaon system in supersymmetric SU(5) model with seesaw induced neutrino masses, Phys. Rev. D 64, 095012 (2001).

[42] A. Romanino and A. Strumia, Electron and muon electric dipoles in supersymmetric scenarios, Nucl. Phys. B622, 73 (2002).

[43] J. R. Ellis, J. Hisano, S. Lola, and M. Raidal, $C P$ violation in the minimal supersymmetric seesaw model, Nucl. Phys. B621, 208 (2002).
[44] J. R. Ellis, J. Hisano, M. Raidal, and Y. Shimizu, Lepton electric dipole moments in nondegenerate supersymmetric seesaw models, Phys. Lett. B 528, 86 (2002).

[45] D. Chang, A. Masiero, and H. Murayama, Neutrino mixing and large $C P$ violation in B physics, Phys. Rev. D 67, 075013 (2003).

[46] J. R. Ellis, J. Hisano, M. Raidal, and Y. Shimizu, A New parametrization of the seesaw mechanism and applications in supersymmetric models, Phys. Rev. D 66, 115013 (2002).

[47] J. Hisano and Y. Shimizu, GUT relation in neutrino induced flavor physics in SUSY SU(5) GUT, Phys. Lett. B 565, 183 (2003).

[48] T. Fukuyama, T. Kikuchi, and N. Okada, Lepton flavor violating processes and muon $\mathrm{g}-2$ in minimal supersymmetric SO(10) model, Phys. Rev. D 68, 033012 (2003).

[49] I. Masina, Lepton electric dipole moments from heavy states Yukawa couplings, Nucl. Phys. B671, 432 (2003).

[50] M. Ciuchini, A. Masiero, L. Silvestrini, S. K. Vempati, and O. Vives, Grand Unification of Quark and Lepton FCNCs, Phys. Rev. Lett. 92, 071801 (2004).

[51] J. Hisano, M. Kakizaki, M. Nagai, and Y. Shimizu, Hadronic EDMs in SUSY SU(5) GUTs with right-handed neutrinos, Phys. Lett. B 604, 216 (2004).

[52] S. T. Petcov, W. Rodejohann, T. Shindou, and Y. Takanishi, The See-saw mechanism, neutrino Yukawa couplings, LFV decays $1(i) \longrightarrow l(j)+$ gamma and leptogenesis, Nucl. Phys. B739, 208 (2006).

[53] F. Deppisch, H. Pas, A. Redelbach, and R. Ruckl, Constraints on SUSY seesaw parameters from leptogenesis and lepton flavor violation, Phys. Rev. D 73, 033004 (2006).

[54] L. Calibbi, A. Faccia, A. Masiero, and S. K. Vempati, Lepton flavour violation from SUSY-GUTs: Where do we stand for MEG, PRISM/PRIME and a super flavour factory, Phys. Rev. D 74, 116002 (2006).

[55] S. T. Petcov and T. Shindou, Charged lepton decays $l(i) \rightarrow l(j)+\gamma$, leptogenesis $C P$-violating parameters and Majorana phases, Phys. Rev. D 74, 073006 (2006).

[56] S. Antusch, E. Arganda, M. J. Herrero, and A. M. Teixeira, Impact of theta(13) on lepton flavour violating processes within SUSY seesaw, J. High Energy Phys. 11 (2006) 090.

[57] J. Hisano and Y. Shimizu, $C P$ violation in $B_{s}$ mixing in the SUSY SU(5) GUT with right-handed neutrinos, Phys. Lett. B 669, 301 (2008).

[58] P. Ko, J. h. Park, and M. Yamaguchi, Sflavor mixing map viewed from a high scale in supersymmetric SU(5), J. High Energy Phys. 11 (2008) 051.

[59] J. Hisano, M. Nagai, and P. Paradisi, Flavor effects on the electric dipole moments in supersymmetric theories: A beyond leading order analysis, Phys. Rev. D 80, 095014 (2009).

[60] F. Borzumati and T. Yamashita, Minimal supersymmetric SU(5) model with nonrenormalizable operators: Seesaw mechanism and violation of flavour and $C P$, Prog. Theor. Phys. 124, 761 (2010).

[61] J. Hisano, M. Nagai, P. Paradisi, and Y. Shimizu, Waiting for $\mu \rightarrow$ e $\gamma$ from the MEG experiment, J. High Energy Phys. 12 (2009) 030. 
[62] M. Hirsch, F. R. Joaquim, and A. Vicente, Constrained SUSY seesaws with a $125 \mathrm{GeV}$ Higgs, J. High Energy Phys. 11 (2012) 105.

[63] T. Moroi and M. Nagai, Probing supersymmetric model with heavy sfermions using leptonic flavor and $C P$ violations, Phys. Lett. B 723, 107 (2013).

[64] D. McKeen, M. Pospelov, and A. Ritz, Electric dipole moment signatures of PeV-scale superpartners, Phys. Rev. D 87, 113002 (2013).

[65] T. Moroi, M. Nagai, and T. T. Yanagida, Lepton flavor violations in high-scale SUSY with right-handed neutrinos, Phys. Lett. B 728, 342 (2014).

[66] W. Altmannshofer, R. Harnik, and J. Zupan, Low energy probes of $\mathrm{PeV}$ scale sfermions, J. High Energy Phys. 11 (2013) 202.

[67] T. Goto, Y. Okada, T. Shindou, M. Tanaka, and R. Watanabe, Lepton flavor violation in the supersymmetric seesaw model after the LHC 8 TeV run, Phys. Rev. D 91, 033007 (2015).

[68] C. Smith and S. Touati, Electric dipole moments with and beyond flavor invariants, Nucl. Phys. B924, 417 (2017).

[69] J. L. Evans, K. Kadota, and T. Kuwahara, Revisiting flavor and $C P$ violation in supersymmetric $S U(5)$ with righthanded neutrinos, Phys. Rev. D 98, 075030 (2018).

[70] Z. Maki, M. Nakagawa, and S. Sakata, Remarks on the unified model of elementary particles, Prog. Theor. Phys. 28, 870 (1962).
[71] B. Pontecorvo, Neutrino experiments and the problem of conservation of leptonic charge, Sov. Phys. JETP 26, 984 (1968).

[72] D. Buttazzo, G. Degrassi, P. P. Giardino, G. F. Giudice, F. Sala, A. Salvio, and A. Strumia, Investigating the nearcriticality of the Higgs boson, J. High Energy Phys. 12 (2013) 089.

[73] E. Bagnaschi, G. F. Giudice, P. Slavich, and A. Strumia, Higgs mass and unnatural supersymmetry, J. High Energy Phys. 09 (2014) 092.

[74] B. C. Allanach, SOFTSUsy: A program for calculating supersymmetric spectra, Comput. Phys. Commun. 143, 305 (2002).

[75] V. Andreev et al. (ACME Collaboration), Improved limit on the electric dipole moment of the electron, Nature (London) 562, 355 (2018).

[76] A. M. Baldini et al. (MEG Collaboration), Search for the lepton flavour violating decay $\mu^{+} \rightarrow \mathrm{e}^{+} \gamma$ with the full dataset of the MEG experiment, Eur. Phys. J. C 76, 434 (2016).

[77] G. F. Giudice, A. Notari, M. Raidal, A. Riotto, and A. Strumia, Towards a complete theory of thermal leptogenesis in the SM and MSSM, Nucl. Phys. B685, 89 (2004).

[78] W. Buchmuller, P. Di Bari, and M. Plumacher, Leptogenesis for pedestrians, Ann. Phys. (Amsterdam) 315, 305 (2005).

[79] K. Hirao and T. Moroi (to be published). 